\title{
Resilience and therapeutic regimen compliance in patients undergoing hemodialysis in hospitals of Hamedan, Iran
}

\author{
Nesa Noghan ${ }^{1}$, Arash Akaberi ${ }^{2,3}$, Sepideh Pournamdarian ${ }^{4}$, Elham Borujerdi $^{5}$, Sima Sadat Hejazi $^{6}$
}

${ }^{1}$ M.Sc. of Critical Care Nursing, Asadabad Faculty of Medical Sciences, Hamedan University of Medical Sciences and Health Services, Hamedan, Iran

${ }^{2}$ M.Sc. of Biostatistics, Sabzevar University of Medical Sciences, Sabzevar, Iran

${ }^{3}$ Biostatistician at McGill University, Montreal, Canada

${ }^{4}$ M.Sc. of General Psychology, Roozbeh Mandegar Psychological Counseling Center, Tehran, Iran

${ }^{5}$ B.Sc. of Nursing, Atiye Hospital, Hamedan, Iran

${ }^{6}$ M.Sc. of Critical Care Nursing, Faculty Member, Nursing Department, Bojnurd Faculty of Nursing and Midwifery, North Khorasan University of Medical Sciences, Bojnurd, Iran

\section{Type of article: Original}

\begin{abstract}
Background: The relationship between resilience, one of the important issues in mental health, and therapeutic regimen compliance, the key in the successful management of chronic disease such as chronic kidney disease, is unclear.

Objective: The aim of this study was to determine the relationship between resilience and therapeutic regimen compliance in ESRD patients, undergoing hemodialysis.

Methods: In this cross-sectional study, 107 hemodialysis patients referred to Besat and Shahid Beheshti Hospitals of Hamedan, Iran were selected through systematic sampling method from January to April 2013. The tool was Connor-Davidson Resilience Scale (CD-RISC). The criteria for compliance were: mean of interdialytic weight gain less than $5.7 \%$ to the dry weight, serum potassium $5.5 \mathrm{mEq} / \mathrm{L}$ or less, serum phosphorus of $6 \mathrm{mg} / \mathrm{dL}$ or less and no more than 3 absences in dialysis sessions. For data analysis, statistical tests such as independent ttest and Logistic regression were performed.

Results: Fifty-seven (53.3\%) patients were males and the mean age of subjects was $49.96 \pm 17.39$ years. The difference in the mean scores of resilience between compliance or non-compliance patients was statistically significant $(\mathrm{p}=0.032)$. Only resilience and age were significant factors related to regimen compliance. In those subjects with greater resilience for 1 score, the chance of compliance with the therapeutic regimen would be $5.4 \%$ higher ( $\mathrm{OR}=1.054$, CI 95\%: 1.01-1.103). In addition, the elderly patients were more likely to comply with the regimen $(\mathrm{OR}=1.072$, CI $95 \%: 1.033,1.113)$.

Conclusion: According to the results, the patients with greater scores of resilience were more likely to comply with the therapeutic regimen.
\end{abstract}

Keywords: Hemodialysis, Resilience, Patient compliance

\section{Introduction}

Chronic kidney disease (CKD) is associated with a decrease in renal function due to age that is aggravated by factors such as high blood pressure, diabetes, obesity or primary renal disorders. This decline in kidney function is progressive and irreversible, so for prevention of severe complications such as uremia, patients must undergo renal

\section{Corresponding author:}

Sima Sadat Hejazi, Nursing Department, Bojnurd Faculty of Nursing and Midwifery, North Khorasan University of Medical Sciences, Bojnurd, Iran. Tel: +985832297096, Email: s.hejazi@nkums.ac.ir

Received: September 30, 2017, Accepted: April 07, 2018, Published: May 2018

iThenticate screening: April 05, 2018, English editing: April 21, 2018, Quality control: May 12, 2018

This article has been reviewed / commented by three experts

Funding / research project approval: 91/60/1912

Ethics approval: $91 / \mathrm{p} / 539$

(C) 2018 The Authors. This is an open access article under the terms of the Creative Commons Attribution-NonCommercialNoDerivs License, which permits use and distribution in any medium, provided the original work is properly cited, the use is non-commercial and no modifications or adaptations are made. 
replacement therapies such as hemodialysis and renal transplantation $(1,2)$. It is estimated that more than two million people in the world are undergoing maintenance hemodialysis treatment (3). In Iran, 15\% is added annually to hemodialysis patients (4). Regularly-scheduled hemodialysis and complex therapeutic regimens impose a heavy burden on patients (5) and severely affects all aspects of individual, family and social life of them (3). Success in controlling CKD depends on compliance with the treatment (6). The results of studies show that dietary regimen noncompliance, which includes intake of medications, fluid and dietary restrictions, effectively increases the risk of hospitalization and mortality (7). Dietary noncompliance and malnutrition indirectly affect the symptoms of depression and are associated with poorer therapeutic outcomes (8). The results of studies show that when the complexity and length of the therapeutic regimen are greater, the likelihood of its nonadherence also increases (9). Due to the prevalence of therapeutic non-compliance, compliance with treatment regimen has been the subject of many clinical investigations since 1970. In hemodialysis patients, non-compliance with the therapeutic regimen is common, and $20 \%$ to $86 \%$ of these patients have shown non-compliance in one or more aspects of therapeutic regimens (10) but the median is near to $50 \%$. Self-reported findings show that noncompliance with fluid restrictions regimen is $30 \%$ to $74 \%$ but non-adherence levels indicated by interdialytic weight gain had a wide range, from $10 \%$ to $60 \%$. Noncompliance of potassium restricted regimen and phosphorus-binding medications are $2 \%$ to $39 \%$ and $19 \%$ to $57 \%$ respectively (11). Currently, various studies have focused on identifying the predictors of therapeutic regimen compliance in patients with chronic kidney disease. The results of the studies show that motivation by the staff, the level of knowledge and social support is associated with the compliance of the therapeutic regimen. However, in general, studies still fail to find a stable relationship between demographic and psychosocial factors and therapeutic regimen noncompliance (9). One of the important issues in mental health and psychology is resilience. Resilience means the ability to adapt to difficult situations and provide flexible responses to daily stresses (12). Resilience can develop a kind of ability and control in the person that enables him to overcome some of the physical and economic burdens and psychosocial consequences of the disease (13). As mentioned, chronic kidney disease and hemodialysis treatment cause many psychological stresses on the patient and since resilience is the ability to cope with traumatic situations (12-14), it is thought that resilient patients have a greater acceptance of the disease, resulting in higher compliance with therapeutic regimens. Due to the high level of therapeutic regimen noncompliance in Iranian hemodialysis patients, and also a limitation of research on the determination of resilience among hemodialysis patients, we investigate the relationship between resilience and therapeutic regimen compliance in patients undergoing hemodialysis.

\section{Material and Methods \\ 2.1. Study design and patients}

This cross-sectional study was done in Besat and Shahid Beheshti Hospitals of Hamedan, Iran from January to April 2013. All the hemodialysis patients referred to Besat and Shahid Beheshti Hospitals of Hamedan, Iran were study population. The inclusion criteria were as follows: age older than 18 years, a six-month interval (at least) since the first hemodialysis session, willingness to participate in the study, without acute physical illness or non-pleasant events during six months prior to the study (events such as death or severe illness of family members or close friends), and exclusion criteria were known mental or cognitive disorder and hospitalization. Due to the limitations of similar studies, we performed a pilot to obtain the sample size. First, 60 patients were randomly included, and then with $80 \%$ power and $95 \%$ confidence, 107 patients were selected through systematic sampling method to be enrolled in the study from patients undergoing hemodialysis in Shahid Beheshti and Besat Hospitals of Hamedan, Iran. The total number of patients with the inclusion criteria was 150. After a random sampling of 60 patients for determining the sample size, a sampling frame consisting of 90 patients was formed, then the first patient was randomly selected and from the number of this patient with the sampling interval of 2, other patients were selected randomly.

\subsection{Measurements}

The tool used in this study was a questionnaire with three parts, of which first two parts were prepared by the researchers after reading resources provided. The first part of the questionnaire included ten questions about demographic data such as age, sex, income level, education level and marital status; the second part included six questions about disease such as disease duration, hemodialysis duration, the number of dialyses per week. In order to determine content validity of these two parts, the questionnaire was presented to the eight faculty members of North Khorasan University of Medical Sciences and, two hemodialysis patients and two nursing students. The third part was the Connor-Davidson Resilience Scale (CD-RISC) which consists of twenty-five items, and each item rates from 0 to 4 (5 points), higher scores reflect greater resilience (14). The questionnaires were completed in collaboration with the researchers during hemodialysis. The patients' mean levels of potassium and phosphorus in 
the past three months were recorded; also, the interdialytic weight gain was measured between the two periods of dialysis. The criteria for the therapeutic regimen compliance were as follows: 1) mean of inter dialytic weight gain less than $5.7 \%$ to the dry weight, 2) serum potassium $5.5 \mathrm{mEq} / \mathrm{L}$ or less, 3) serum phosphorus of $6 \mathrm{mg} / \mathrm{dL}$ or less, and 4) no more than 3 absences in dialysis sessions. Failure to adhere to even one of these criteria was considered as non-compliance $(5,6,11)$. After determining the patient's compliance with therapeutic regimen and resilience, the correlation between these two variables was analyzed.

\subsection{Validity and reliability}

CD-RISC includes 25 items and is graded based on the five-point Likert scale (zero: completely false; five: completely true). In this scale, the minimum score of the individual's resilience is zero and the maximum is 100 (14). CD-RISC is a validated and reliable scale which can be translated into several languages and used in different groups $(12,13)$. For the first time, Mohammadi adapted this scale in Iran (12). In this study, the reliability of the CD-RISC was estimated as 0.76 by Cronbach's alpha coefficient.

\subsection{Data collection}

After obtaining the written consents and introducing the researcher to the study environment, the questionnaires were completed by all patients, who met the inclusion criteria during hemodialysis. Based on the information in patients' medical records, predialysis serum levels of potassium and phosphorus, as well as the interdialytic weight gain and dry weight, were recorded in the past three months for each patient. In order to collect the data during the investigation, the researcher was present in the morning, evening, and at night shifts.

\subsection{Ethical consideration}

The subjects were ensured about the confidentiality of the data, and consent was obtained from the respective participants. This study was approved by the ethical committee of North Khorasan University of Medical Sciences (research code $91 / \mathrm{p} / 539$ ).

\subsection{Statistical analysis}

For the comparison of the mean between the patients, a two-tailed independent t-test was applied. Chi-square test was used for the comparison of qualitative variables between the patients. Logistic regression with conditional backward method was used for the prediction of Odds Ratio (OR) of accepted regimen compliance in terms of resilience, age, gender, education, employment, and dialysis duration (years). Statistical analysis was performed using SPSS (SPSS Inc., Chicago, Illinois, USA) version 17. P-value less than 0.05 was considered statistically significant.

\section{Results}

In this study, 107 patients were assessed, 57 (53.3\%) patients were males and the mean age of them was $49.96 \pm 17.39$ years. Other demographic and clinical characteristics are provided in Table 1 . Of all the subjects, only $25(23.4 \%)$ cases had therapeutic regimen compliance, and $82(76.6 \%)$ patients had non-compliance. Other results of therapeutic regimen compliance are provided in Table 2 . The independent-samples t-test showed that there was no association between the two genders in terms of regimen compliance $(\mathrm{p}=0.441)$. The mean score of resilience was $75.04 \pm 14.54$. Among subjects who adhered to the regimens, the mean score of resilience was $80.48 \pm 15.71 \mathrm{vs}$. $73.38 \pm 13.84$ for those who did not adhere to the regimens. The difference in the mean scores of resilience between patients with compliance and without compliance was statistically significant $(\mathrm{p}=0.032)$. To predict compliance or non-compliance with the therapeutic regimen, we used resilience variables such as age, gender, education, employment, and dialysis duration (years). By calculating conditional logistic regression, it was concluded that only resilience and age were significant factors related to regimen compliance. According to the results, in those subjects with greater resilience for 1 score, the chance of compliance with the therapeutic regimen would be $5.4 \%$ higher $(\mathrm{OR}=1.054$, CI 95\%: 1.01-1.103). In addition, elderly patients were more likely to comply with the dietary regimen, and the high level of therapeutic regimen compliance increased to 1.072 over one year $(\mathrm{OR}=1.072 \mathrm{CI} 95 \%: 1.033$, 1.113) (Table 3). 
Table 1. Demographic and clinical characteristics of patients undergoing hemodialysis $(\mathrm{n}=107)$

\begin{tabular}{|c|c|c|}
\hline \multicolumn{2}{|l|}{ Characteristic } & Values; n (\%) / Mean \pm SD \\
\hline \multirow{2}{*}{ Gander } & Male & $57(53.3)$ \\
\hline & Female & $50(46.7)$ \\
\hline \multirow[t]{4}{*}{ Marital status } & Married & $77(72)$ \\
\hline & Single & $16(15)$ \\
\hline & Widow & $12(11.2)$ \\
\hline & Divorced & $2(1.9)$ \\
\hline \multirow[t]{5}{*}{ Education } & Literacy & $12(11.2)$ \\
\hline & Primary School & $34(31.8)$ \\
\hline & Secondary School & $16(15)$ \\
\hline & Diploma & $25(23.4)$ \\
\hline & Collegiate & $20(18.7)$ \\
\hline \multirow[t]{8}{*}{ Occupational status } & Unemployed & $13(12.1)$ \\
\hline & Employee & $10(9.3)$ \\
\hline & Housekeeper & $41(38.3)$ \\
\hline & Farmer & $2(1.9)$ \\
\hline & Worker & $4(3.7)$ \\
\hline & Others & $14(13.1)$ \\
\hline & Retired & $9(8.4)$ \\
\hline & Disabled & $14(13.1)$ \\
\hline \multirow[t]{3}{*}{ Income sufficiency } & Yes & $15(14)$ \\
\hline & No & $38(38)$ \\
\hline & Somewhat & $54(50.5)$ \\
\hline \multirow{2}{*}{ Dialysis frequency (per week } & 2 & $35(32.7)$ \\
\hline & 3 & $72(67.3)$ \\
\hline \multirow[t]{2}{*}{ History of renal transplantation } & Yes & $11(10.3)$ \\
\hline & No & $96(89.7)$ \\
\hline \multirow[t]{2}{*}{ Other disease } & Yes & $60(56.1)$ \\
\hline & No & $47(43.9)$ \\
\hline \multicolumn{2}{|l|}{ Duration of dialysis (year) } & $2.49 \pm 0.87$ \\
\hline
\end{tabular}

Table 2. Compliance of patients undergoing hemodialysis

\begin{tabular}{|l|l|}
\hline Compliance & $\mathrm{n}(\%)$ \\
\hline Potassium restricted regimen & $87(81.3)$ \\
\hline Phosphorus restricted and medication & $54(50.5)$ \\
\hline Fluid restricted regimen & $53(49.5)$ \\
\hline Attendance in dialysis sessions & $105(98.1)$ \\
\hline Total & $25(23.4)$ \\
\hline
\end{tabular}

Table 3. The findings of logistic regression by conditional method of prediction of compliance by therapeutic regimen via resiliency and demographic variables

\begin{tabular}{|l|l|l|l|l|l|}
\hline Variables & $\mathrm{B}$ & $\mathrm{p}$-value & OR & \multicolumn{2}{|c|}{$95 \%$ CI for OR } \\
\cline { 5 - 6 } & & & & Lower & Upper \\
\hline Resilience & 0.053 & 0.023 & 1.054 & 1.007 & 1.103 \\
\hline Age & 0.069 & $<0.001$ & 1.072 & 1.033 & 1.113 \\
\hline Constant & -9.077 & $<0.001$ & $\mathrm{p}<0.001$ & & \\
\hline
\end{tabular}

OR: Odds ratio; CI: Confidence interval

\section{Discussion}

This study aimed to determine the relationship between resilience and therapeutic regimen compliance in patients undergoing hemodialysis. Our main finding is that resilience is related to the therapeutic regimen compliance and in patients with greater resilience scores; the chance of therapeutic regimen compliance is increased. In this study, we found that the therapeutic regimen compliance in hemodialysis patients is low while in the Medeiros et al study, 
$40.7 \%$ of patients are noncompliant. This difference may be related to the lower mean age of patients in our study and the use of the self-report tool in the Medeiros study (3). The results of Melon et al. indicated that $62 \%$ of the patients were noncompliant in at least one of the domains of therapeutic regimen, the patients in our study were in a worse case in terms of compliance. This may still be due to the higher mean age of the subjects in their study (9). The most important causes of therapeutic regimen non-compliance of dialysis patients according to studies in Iran are factors such as psychological problems and especially depression, which plays an important role in the isolation and dismay of the individual and as a consequence of therapeutic regimen non-compliance. Other factors such as the lack of awareness and attitude of dialysis patients about their treatment and lack of adequate social support are also involved in this regard (4). According to a report by Rambod et al, the results of the studies indicate that the range of potassium restricted regimen compliance is between $60 \%$ and $65 \%$; and the results of their study on potassiumrestricted regimen compliance in Iranian patients were 5.94\%. Adoption of potassium restricted regimen compliance in our study is $81.3 \%$, which is similar to Rambod's results. This significant difference in potassium restricted regimen compliance of Iranian patients may be due to differences in the dietary habits of Iranian patients with others (11). Studies showed that the range of adherence to the medication (phosphorus bindings) and phosphorus restricted dietary are $50.5 \%$ (11), the results of the present study are consistent with this range but the results of Rambod et al showed that $74.5 \%$ of the patients had medication compliance, which is different from the results of our study. The mean age of the patients assessed by Rambod et al was more than that of our patients, and one of the reasons for this difference might be this (11). Khalil et al. and Staji et al reported that almost half of the patients in terms of serum phosphorus status are within acceptable range, which is similar to the results of this study $(6,8)$. The lack of knowledge about phosphorus-containing foods and the importance of using phosphorus-binding medications and the lack of education about the need for changes in individual habits can be related to this problem.

In the present study, the compliance of potassium restricted regimen was better than the phosphorus restricted regimen and medication regimen compliance; these results are similar to those of Rambod and Park $(11,15)$. Serum potassium can be controlled by dietary compliance only, but in order to prevent the increase in blood phosphorus levels, both medication and dietary regimen compliance are needed. Therefore, the higher level of non-compliance in this area than the potassium restricted regimen in the present study as well as in other studies can be related to this problem. One of the other reasons is the early onset of high levels of serum potassium symptoms in patients, such as gastrointestinal symptoms which are rapidly relieved by dietary compliance. It is also possible that the restriction of high-potassium foods is easier than phosphorus-containing foods since potassium-rich foods are mostly vegetables and fruits (15). In the present study, older patients were more compliant with the therapeutic regimen. Khalil et al and Park et al. found similar results $(8,15)$. The reason is likely that the older patients worry about their health more than younger patients. Younger people, due to more social relationships, are also more likely to consume food in restaurants, and parties etc., which can lead to noncompliance. The results of this study showed that resilience is related to the therapeutic regimen compliance regimen, which is similar to Medeiros' study (3). Also, the results of $\mathrm{Ma}$ et al indicated a significant positive relationship between health-promoting behaviors and resilience (16). Therapeutic regimen compliance can be a byproduct of resilience. Grunberg defined resilience as the unique ability of persons to prevent, limit, and overcome the harmful effects of difficult conditions, such as chronic disease (17). We used the medical records from the past three months and this might be one of our study limitations, also the serum markers such as potassium and phosphorus might be affected by dialysis.

\section{Conclusions}

According to the results of this study, compliance in the patients is low. Also, compliance is related to resilience, and patients with a greater score of resilience were more likely to comply with the therapeutic regimen. The results indicate that the issue of therapeutic regimen compliance in hemodialysis patients still needs attention. Also, according to the findings of this study which indicates the relationship between resiliency and regimen compliance, it is necessary to pay attention to the dimensions of the mental health of patients with chronic illness. It is suggested to investigate the promoting factors of resilience and the effect of them on compliance in future studies.

\section{Acknowledgments:}

This research was undertaken at the Addiction and Behavioral Sciences Research Center and was financially supported by North Khorasan University of Medical Sciences. The authors wish to thank them for their support and gratefully acknowledge the collaboration of all the hemodialysis patients.

\section{Conflict of Interest:}

There is no conflict of interest to be declared. 


\section{Authors' contributions:}

All authors contributed to this project and article equally. All authors read and approved the final manuscript.

\section{References:}

1) Hill NR, Fatoba ST, Oke JL, Hirst JA, O'Callaghan CA, Lasserson DS, et al. Global prevalence of chronic kidney disease - A systematic review and meta-analysis. PLoS One. 2016; 11(7): e0158765. doi: 10.1371/journal.pone.0158765 PMID: 27383068 PMCID: PMC4934905.

2) Moreira JM, Bouissou Morais Soares CM, Teixeira AL, Simões E Silva AC, Kummer AM. Anxiety, depression, resilience and quality of life in children and adolescents with pre-dialysis chronic kidney disease. Pediatr Nephrol. 2015; 30(12): 2153-62. doi: 10.1007/s00467-015-3159-6. PMID: 26210984.

3) Freire de Medeiros CM, Arantes EP, Tajra RD, Santiago HR, Carvalho AF, Libório AB. Resilience, religiosity and treatment adherence in hemodialysis patients: a prospective study. Psychol Health Med. 2017; 22(5): 570-7. doi: 10.1080/13548506.2016.1191658. PMID: 27249545.

4) Hadian Z, Rafiee Vardanjani L, Barimnejad L. The most important causes of non-adherence in patients on dialysis. Clin Exc. 2016; 5(2): 84-97.

5) Hejazi SS, Nikbakht Sh, Nasiri Zarrin Ghabaee D, Akaberi A, Nazari Sheyhaki A. Hope in caregivers and hemodialysis patients. Journal of North Khorasan University of Medical Sciences. 2014; 6(4): 807-17.

6) Estaji Z, Hejazi S, Tabarraie Y, Saedi M. The Effects of training through text messaging via cell phones on the compliance of patients undergoing hemodialysis. Journal of North Khorasan University of Medical Science. 2016; 8 (2): 203-13. doi: 10.18869/acadpub.jnkums.8.2.203.

7) Matteson ML, Russell C. Interventions to improve hemodialysis adherence: a systematic review of randomized-controlled trials. Hemodial Int. 2010; 14(4): 370-82. doi: 10.1111/j.1542-4758.2010.00462.x. PMID: 20796047.

8) Khalil AA, Frazier SK, Lennie TA, Sawaya BP. Depressive symptoms and dietary adherence in patients with end-stage renal disease. J Ren Care. 2011; 37(1): 30-9. doi: 10.1111/j.1755-6686.2011.00202.x.

9) Mellon L, Regan D, Curtis R. Factors influencing adherence among Irish haemodialysis patients. Patient Educ Couns. 2013; 92(1): 88-93. doi: 10.1016/j.pec.2013.01.023. PMID: 23481215.

10) Gerbino G, Dimonte V, Albasi C, Lasorsa C, Vitale C, Marangella M. Adherence to therapy in patients on hemodialysis. G Ital Nefrol. 2011; 28(4): 416-24. PMID: 21809311.

11) Rambod M, Peyravi H, Shokrpour N, Sareban MT. Dietary and fluid adherence in Iranian hemodialysis patients. Health Care Manag (Frederich). 2010; 29(4): 359-64. doi: 10.1097/HCM.0b013e3181fa0691. PMID: 21045589.

12) Ahangarzade rezaei S, Rasoli M. Psychometric properties of the Persian version of conner- Davidson resilience scale in adolescence with cancer. The Journal of Urmia Nursing and Midwifery Faculty. 2015; 13(9): 739-47.

13) Boell JE, Silva DM, Hegadoren KM. Sociodemographic factors and health conditions associated with the resilience of people with chronic diseases: a cross sectional study. Rev Lat Am Enfermagem. 2016; 24: e2786. doi: 10.1590/1518-8345.1205.2786. PMID: 27598377, PMCID: PMC5016006.

14) Conner KM, Davidson JR. Development of a new resilience scale: The Conner- Davidson Resilience Scale (CD-RISC). Depress Anxiety. 2003; 18(2): 76-83. doi: 10.1002/da.10113. PMID: 12964174.

15) Park KA, Choi-Kwon S, Sim YM, Kim SB. Comparison of dietary compliance and dietary knowledge between older and younger Korean hemodialysis patients. J Ren Nutr. 2008; 18(5): 415-23. doi: 10.1053/j.jrn.2008.04.004.

16) Ma LC, Chang HJ, Liu YM, Hsien HL, Lo L, Lin MY, et al. The relationship between health-promoting behaviors and resilience in patients with chronic kidney disease. Scientific World Journal. 2013; 2013: 124973. doi: 10.1155/2013/124973. PMID: 23589703, PMCID: PMC3621294.

17) Grünberg J. The impact of logistic and resilience on compliance. Pediatr Nephrol. 2005; 20(12): 1823-4. doi: 10.1007/s00467-005-2041-3. PMID: 16208533. 